\title{
Predicted Realization of Cubic Dirac Fermion in Quasi-One-Dimensional Transition-Metal Monochalcogenides
}

\begin{abstract}
Qihang Liu* and Alex Zunger
Renewable and Sustainable Energy Institute, University of Colorado, Boulder, Colorado 80309, USA (Received 30 November 2016; revised manuscript received 13 February 2017; published 9 May 2017)

We show that the previously predicted "cubic Dirac fermion," composed of six conventional Weyl fermions including three with left-handed and three with right-handed chirality, is realized in a specific, stable solid state system that has been made years ago, but was not appreciated as a "cubically dispersed Dirac semimetal" (CDSM). We identify the crystal symmetry constraints and find the space group $\mathrm{P}_{3} / \mathrm{m}$ as one of the two that can support a CDSM, of which the characteristic band crossing has linear dispersion along the principle axis but cubic dispersion in the plane perpendicular to it. We then conduct a material search using density functional theory, identifying a group of quasi-one-dimensional molybdenum monochalcogenide compounds $\mathrm{A}^{\mathrm{I}}\left(\mathrm{MoX}^{\mathrm{VI}}\right)_{3}\left(\mathrm{~A}^{\mathrm{I}}=\mathrm{Na}, \mathrm{K}, \mathrm{Rb}, \mathrm{In}, \mathrm{Tl} ; \mathrm{X}^{\mathrm{VI}}=\mathrm{S}, \mathrm{Se}, \mathrm{Te}\right)$ as ideal CDSM candidates. Studying the stability of the $\mathrm{A}(\mathrm{MoX})_{3}$ family reveals a few candidates such as $\mathrm{Rb}(\mathrm{MoTe})_{3}$ and $\mathrm{Tl}(\mathrm{MoTe})_{3}$ that are predicted to be resilient to Peierls distortion, thus retaining the metallic character. Furthermore, the combination of one dimensionality and metallic nature in this family provides a platform for unusual optical signature-polarization-dependent metallic vs insulating response.
\end{abstract}

DOI: 10.1103/PhysRevX.7.021019

\section{INTRODUCTION}

The crossing on energy bands in complex materials showing dense manifold of states is a ubiquitous effect routinely reported in the past $\sim 50$ years in countless publications, a visual effect often referred to as "band spaghetti." For a long time, such crossings have been known to result from specific space-group symmetries $[1,2]$, involving various band dispersion $E(\boldsymbol{k})$ with powers $k^{n}$ and characterized by a band degeneracy $g(\boldsymbol{k})$ at the crossing wave vector $\boldsymbol{k}$. With the renewed interest in materials with strong spin-orbit coupling (SOC), it recently became clear that such band-crossing points could carry interesting information on the topological behavior of the system, leading to specific behaviors of surface or edge states in reduced dimensions [3-5]. In SOC systems respecting time-reversal symmetry, the degeneracy of multiband crossings in momentum space could be $g(\boldsymbol{k})=2,3,4,6$, and 8 , and the dispersion power $k^{n}$ with $n=1,2,3$ at the crossing point could be linear, quadratic, or cubic, respectively. Crossings between conduction and valence bands with linear dispersion in at least one $k$-space direction and $g=2$-fold degeneracy correspond to Weyl semimetal (WSM) [3,6-10] and $g=4$-fold degeneracy to

*qihang.liu85@gmail.com

Published by the American Physical Society under the terms of the Creative Commons Attribution 4.0 International license. Further distribution of this work must maintain attribution to the author(s) and the published article's title, journal citation, and DOI.
Subject Areas: Condensed Matter Physics,

Materials Science
Dirac semimetal (DSM) [4,11-16]. The remaining degeneracies at band-crossing points $g=3,6$, and 8 correspond to quasiparticles without analogous states in the standard model of particle physics [5,17-19]. The latter respects Poincare symmetry and has but three fermion types-Weyl, Dirac, and Majorana.

In general, Dirac or Weyl band crossings in bulk solids could be accompanied by different dispersion powers in different directions, as constrained by their crystal symmetries. At these band crossings (termed "Dirac or Weyl points"), the dispersion along the principle rotation axis $(c)$ is linear, whereas the dispersion along the $a-b$ plane could be either linear $(n=1)$, quadratic $(n=2)$, or cubic $(n=3)$. A Weyl point with in-plane dispersion power $n$ carries a Chern number $n$ or $-n$, corresponding to a degeneracy of $n$ conventional Weyl fermions (left- or right-handed), all with the same chirality [7]. In contrast, a Dirac point (DP) with specific $n$ has zero net Chern number and is a $2 n$-fold degenerate Weyl fermion with half left-handed and half righthanded chirality $[15,20,21]$. Such Weyl and Dirac fermions with high-order dispersions, caused by crystalline symmetry in solids, do not have counterparts in high-energy physics. While the surface states of DSM are not topologically protected (unlike those of TI and WSM), such quadratic $(n=2)$ and cubic Dirac fermions $(n=3)$, especially the latter, are interesting relative to the conventional $n=1$ Dirac fermions: Their distinguishing features (as discussed in Sec. III D) include creation of special WSM with multiple Fermi arcs, characteristic quantum transport signatures, quantum criticality, and phase transitions. 
In addition to the efforts to define and classify such specific "new fermion" band crossings induced by crystalline symmetries and topology in condensed-matter systems [5], an important challenge is to systematically identify material realization of such unusual fermions. We summarize all the different types of fermions in solid-state physics, classified by the degree of band degeneracy $(g)$ and the highest power of band dispersion $(n)$, with example materials in Appendix A. Whereas quadratic dispersion has been predicted to exist in $\mathrm{SrSi}_{2}$ [22] $(g=2)$, bandinverted $\alpha-\operatorname{Sn}(g=4)$ [23], and $\operatorname{PdSb}_{2}(g=6)$ [5], cubic dispersion, expected to exist both in Weyl [7] and Dirac semimetals [15], has not been realized as yet in any material candidates. In part, the difficulty to find such cubically dispersed Dirac semimetals (CDSM) is related to the multitude of nontrivial requirements one needs to impose on such a material search, including appropriate crystal symmetry, angular momentum, and electron filling. Finding such materials by accidental discovery or simple trial-and-error methods would thus be unlikely.

Here, we establish understanding-based design principles for CDSM and use these to deliberately screen the candidates that satisfy such conditions by exhaustively looking through all 230 space groups in $3 \mathrm{D}$. We find that only materials in two space groups, $\mathrm{P}_{3} / \mathrm{m}$ (No. 176) and P6/mcc (No. 192), have the potential to host cubic Dirac fermions. This narrowing down of the possibilities is then followed up by a (yetnonexhaustive) material search of compounds belonging to these two CDSM-hosting space groups using density functional theory (DFT, see Appendix B for computational details). We identify a group of molybdenum transitionmetal chalcogenide compounds $\mathrm{A}^{\mathrm{I}}\left(\mathrm{MoX}^{\mathrm{VI}}\right)_{3}\left(\mathrm{~A}^{\mathrm{I}}=\mathrm{Na}, \mathrm{K}\right.$, $\left.\mathrm{Rb}, \mathrm{In}, \mathrm{Tl} ; \mathrm{X}^{\mathrm{VI}}=\mathrm{S}, \mathrm{Se}, \mathrm{Te}\right)$ with space group $\mathrm{P}_{3} / \mathrm{m}$ as ideal candidates. The structure of this type of compound (Fig. 1) is basically quasi-1D chains $\left(\mathrm{Mo}_{3} \mathrm{X}_{3}\right)^{1}$ running in the direction of the $c$ axis and separated by the hexagonal $\mathrm{A}^{1+}$ framework. Most of these compounds have been synthesized in the 1980s [24], and preliminary electronic structure with linear dispersion along the chain direction has been reported previously [25,26]. Recently, Gibson et al. theoretically proposed this family of compounds as the conventional linearly dispersed DSM [27]. In the present paper, we find that the $\mathrm{A}(\mathrm{MoX})_{3}$ family exhibits (i) one cubically dispersed DP; (ii) three linearly dispersed DPs induced by the nonsymmorphic symmetry within the Brillouin zone (BZ), like those envisioned for $\mathrm{BiO}_{2}$ in a hypothetical unstable structure [11]; and (iii) quadratic DPs and another type of linear DP along the chain direction in the conduction bands. Furthermore, we predict that because of the 1D metallic bands embedded in a semiconductor bulk, $\mathrm{A}(\mathrm{MoX})_{3}$ CDSMs will exhibit polarization-dependent optical response.

To predict realistic CDSM candidates, we systematically studied the stability of these quasi-1D $\mathrm{A}(\mathrm{MoX})_{3}$ compounds under Peierls distortion. DFT results show that some compounds with strong 1D character inevitably experience Peierls distortion, indicating a metal-toinsulator transition below a critical temperature, consistent with the previous transport measurements [28,29]. In contrast, we find that the ground states of some compounds, e.g., $\mathrm{Rb}(\mathrm{MoTe})_{3}$ and $\mathrm{Tl}(\mathrm{MoTe})_{3}$, are immune to Peierls distortion even at low temperature, leading to the retention of the CDSM phase. Another stability issue that will be explored in the future is the resilience of the structure to spontaneous formation of intrinsic defects [such as $\mathrm{A}$ vacancies in $\mathrm{A}(\mathrm{MoX})_{3}$ ] that may shift the Fermi level of the insulating phase.

\section{RESULTS}

\section{A. Design principle for identifying CDSM}

This process involves both crystal symmetry and electronic occupancy conditions. We will first consider the crystal-symmetry requirement for DSM with cubic in-plane dispersions.

Here, we focus on nonmagnetic materials with inversion symmetry $P$ and SOC, which respects time-reversal symmetry $T$ with $T^{2}=-1$. The cubic dispersion at the DP can only appear as the form of fourfold degeneracy occurring in the time-reversal invariant (TRI) $k$ point, whose little group has $\mathrm{C}_{6}$ symmetry [15]. Given that $P$ and $T$ ensure spin degenerate states $\psi(\boldsymbol{k}, \boldsymbol{\sigma})$ and $P T \psi(\boldsymbol{k}, \boldsymbol{\sigma})=\psi(\boldsymbol{k},-\boldsymbol{\sigma})$, we need an extra pair of states $L \psi$ and PTL from $\psi$ and $P T \psi$, where $L$ is a Hermitian symmetry operator that commutes with the Hamiltonian $H$. This goal can be achieved by finding another Hermitian symmetry operator $\mathcal{A}$ to fulfill $\left\{A, A_{P T}\right\} \cap\left\{A_{L}, A_{L P T}\right\}=\emptyset$, where $A$ is the eigenvalue of $\psi$ under $\mathcal{A}$. For the least symmetry required by the system, we first let $L=P$; then the extra degeneracy immediately requires an anticommutation $\{\mathcal{A}, P\}=0$, implying that $\mathcal{A}$ contains a nonsymmorphic symmetry.

Therefore, to establish cubic in-plane dispersion at $g=4$ band crossing, we need at least three symmetry filters: inversion, $\mathrm{C}_{6}$ rotation, and the presence of nonsymmorphic operations such as screw axis or glide reflection. These requirements already exclude most of the space groups and leave only four possibilities: $\mathrm{P}_{3} / \mathrm{m}$ (No. 176), P6/mcc (No. 192), $\mathrm{P}_{3} / \mathrm{mcm}$ (No. 193), and $\mathrm{P}_{3} / \mathrm{mmc}$ (No. 194). All of these space groups have fourfold degeneracy at TRI $k$ points within the $k_{z}=\pi$ plane, forced by screw axis (Nos. 176, 193, and 194) or glide reflection symmetry (No. 192). However, space groups No. 193 and No. 194 have three mirror planes parallel to the $\mathrm{C}_{6}$ axis; these pose extra symmetry conditions that force three high-symmetry lines to be degenerate, and thus, there is no band splitting along these directions [30]. Therefore, such nodes form an infinite network through the BZ, leading to new types of topological semimetals named nodal-line [31-36] and nodal-ring semimetals [30,37], which are distinct from 
DSM and WSM. Therefore, only materials with space groups $\mathrm{P}_{3} / \mathrm{m}$ and $\mathrm{P} 6 / \mathrm{mcc}$ can host cubic Dirac fermions in terms of crystal symmetry (see Appendix C for details).

To further ensure that the leading order of the in-plane dispersion is cubic, the states of the conduction band and valence band at the DP should have opposite eigenvalues of the $\mathrm{C}_{6}$ operator [15], $i$ or $-i$. This means that the four degenerate states at the DP should have an orbital part with angular momentum $l_{z}= \pm 1$, e.g., $p_{x} \pm i p_{y}$ or $d_{x z} \pm i d_{y z}$ components. In addition, the fourfold degenerate DP bunches conduction and valence bands together (half filling), which requires the total number of electrons to be $N_{e} \bmod 4=2$. On the other hand, because of nonsymmorphic symmetry, the materials should have at least two sublattices, indicating an odd number count per formula unit (f.u.).

We summarize all the requirements for CDSM as follows: (i) inversion, (ii) $\mathrm{C}_{6}$ rotation, (iii) nonsymmorphic symmetry, (iv) $l_{z}= \pm 1$ states, and (v) odd number of electrons per f.u. Based on these design principles, we performed a design-principle guided material search and found that a group of $\mathrm{A}(\mathrm{MoX})_{3}$ compounds with space group $\mathrm{P}_{3} / \mathrm{m}$ are ideal candidates for CDSM.

\section{B. Crystal structure and chemical bonding of the $\mathrm{A}(\mathrm{MoX})_{3}$ family}

The quasi-1D structures $\mathrm{A}(\mathrm{MoX})_{3}$ system is derived from the general family, known as "Chevrel clusters" [38], that has been synthesized with extended MoX clusters $\mathrm{Mo}_{6} \mathrm{X}_{8}$. The $\mathrm{Mo}_{6} \mathrm{X}_{8}$ unit can be viewed as a $\mathrm{Mo}_{6}$ octahedral surrounded by eight $\mathrm{X}$ chalcogen atoms, or two $\mathrm{Mo}_{3} \mathrm{X}_{3}$ star-shaped planes capped by two other $\mathrm{X}$ atoms along the $c$ axis $\left(\mathrm{C}_{6}\right.$ rotation axis), as shown in Fig. 1(a). This unit cluster can be expanded along the $c$ axis infinitely and can form a $1 \mathrm{D}$ chain by repeating the $\mathrm{Mo}_{3} \mathrm{X}_{3}$ unit along the $c$ axis, as shown in Figs. 1(b) and 1(c). Such an extension provides a transition between a "molecule" and a chainlike structure, with each equidistant unit of the chain forming an equilateral triangle by three Mo atoms. The monovalent cation element A (alkali or In, Tl) forms linear chains between the $\mathrm{Mo}_{3} \mathrm{X}_{3}$ units, as shown in Fig. 1(e).

The intratriangle (within the $a-b$ plane) Mo-Mo bond lengths are 2.64-2.65 $\AA$ for all the $\mathrm{A}(\mathrm{MoX})_{3}$ compounds considered here, while the intertriangle Mo-Mo bond ranges from 2.69 to $2.76 \AA$. These bond lengths are all well below twice the atomic radius of Mo (2.01 $\AA$ ), indicating a strong Mo-Mo interaction. In addition to the ordinary ionic or valent bonds (e.g., Mo-X), the existence of an Mo-Mo interaction leads to delocalized electrons along the chain and complicates the overall bonding types of Mo atoms. As a result, the average valence state of Mo can be a fractional number, offering possibilities to have an odd number of electrons per f.u. Considering $\mathrm{A}(\mathrm{MoX})_{3}$ with alkali atom $\mathrm{A}\left(\mathrm{s}^{1}\right), \operatorname{Mo}\left(\mathrm{d}^{5} \mathrm{~s}^{1}\right)$, and $\mathrm{X}\left(\mathrm{s}^{2} \mathrm{p}^{4}\right)$ and that only the Mo atom can be multivalent, the valence state on the Mo atom is thus +1.67 . The total electrons per f.u. is an odd number, 37.

In addition to the odd number of electron filling, we have also identified this family of compounds as a CDSM because of its reported space group being nonsymmorphic $\mathrm{P}_{3} / \mathrm{m}$ (No. 176) [24]. The structure contains 12 symmetry operations: identity; inversion; screw axis operation
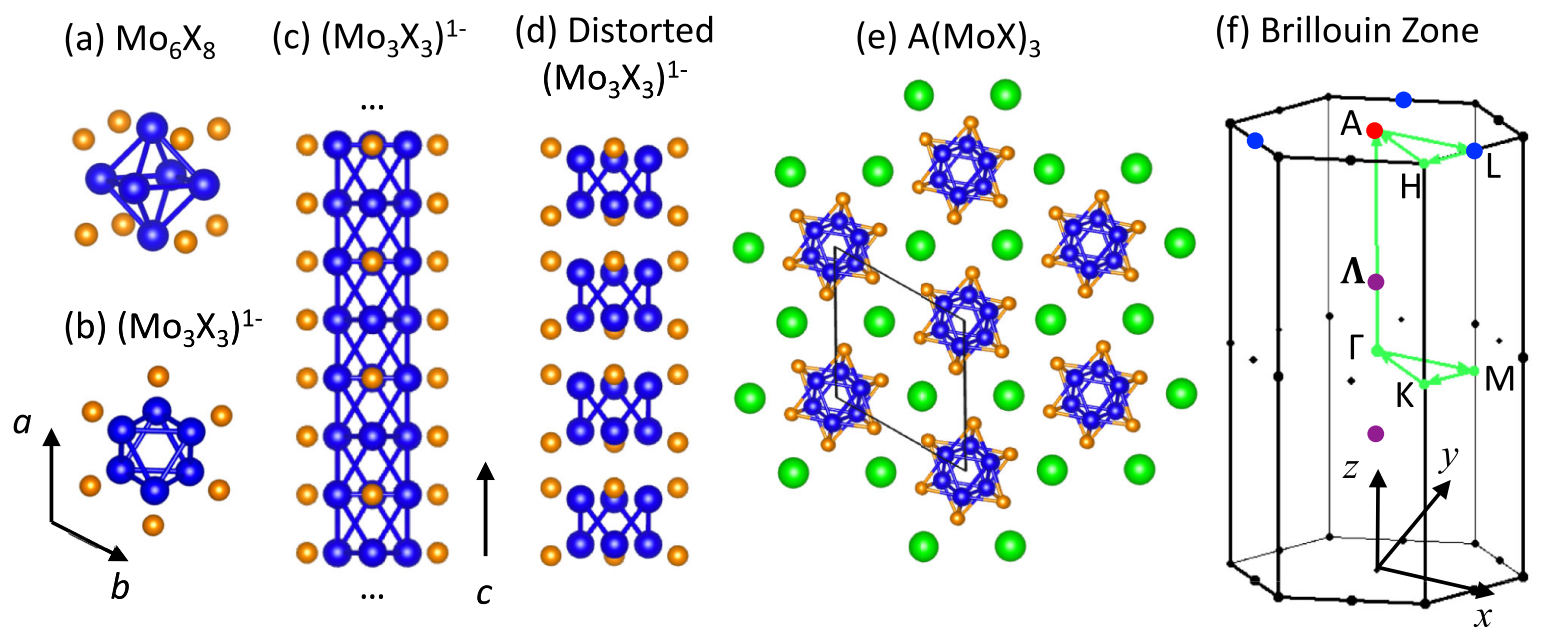

FIG. 1. (a) Structure of the $\left(\mathrm{Mo}_{6} \mathrm{X}_{8}\right)$ cluster unit with a $\mathrm{Mo}_{6}$ octahedral surrounded by a cubic $\mathrm{X}_{8}$ cage. (b) Top and (c) side view of the quasi-1D chain $\left(\mathrm{Mo}_{3} \mathrm{X}_{3}\right)^{1-}$. (d) Side view of the quasi-1D chain $\left(\mathrm{Mo}_{3} \mathrm{X}_{3}\right)^{1-}$ with Peierls distortion. (e) Crystal structure of the cluster compound $\mathrm{A}(\mathrm{MoX})_{3}$. The blue, orange, and green balls denote Mo, X, and A atoms, respectively. (f) Hexagonal Brillouin zone and the high-symmetry $k$ path for band structure calculation. The dots mark the inequivalent Dirac points (one A point, three L points, and two $\boldsymbol{\Lambda}$ points representing $\boldsymbol{\Lambda}_{1}$ and $\boldsymbol{\Lambda}_{2}$ ) in undistorted structure. 
TABLE I. Different types of Dirac points and their physical properties in $\mathrm{Tl}(\mathrm{MoTe})_{3}$. Note that the energy of $\mathrm{DP}$ is related to the Fermi level, and $w=e^{i \pi / 6}$.

\begin{tabular}{lcccccc}
\hline \hline $\begin{array}{l}\text { Wave vector } \\
\text { of DP }\end{array}$ & $\begin{array}{c}\text { Multiplicity } \\
\text { in BZ }\end{array}$ & $\begin{array}{c}\text { Energy } \\
(\mathrm{eV})\end{array}$ & $\begin{array}{c}\text { In-plane } \\
\text { dispersion }\end{array}$ & $\begin{array}{c}\text { Little group } \\
\text { of DP }\end{array}$ & $\begin{array}{c}\text { Inversion } \\
\text { operator P }\end{array}$ & $\begin{array}{c}\text { Rotation } \\
\text { eigenvalue }\end{array}$ \\
\hline $\mathrm{A}(0,0,0.5)$ & 1 & -0.226 & Cubic & $\mathrm{C}_{6 h}$ & $\pm \sigma_{x}$ & $\{i,-i,-i, i\}$ \\
$\mathrm{L}(0.5,0,0.5)$ & 3 & 0.007 & Linear & $\mathrm{C}_{2 h}$ & $\pm \sigma_{x}$ & $\{i,-i,-i, i\}$ \\
$\boldsymbol{\Lambda}_{1}(0,0,0.154)$ & 2 & 0.294 & Quadratic & $\mathrm{C}_{6}$ & $\pm \sigma_{z}$ & $\left\{w, w^{5}, w^{-1}, w^{-5}\right\}$ \\
$\boldsymbol{\Lambda}_{2}(0,0,0.136)$ & 2 & 0.328 & Linear & $\mathrm{C}_{6}$ & $\pm \sigma_{z}$ & $\left\{w, \mathrm{w}^{-1}, i,-i\right\}$ \\
\hline \hline
\end{tabular}

$\left\{\mathrm{C}_{6} \mid(0,0,1 / 2)\right\}$, which is a sixfold rotation about the $c$ axis followed by a fractional lattice translation $c / 2$; and their combinations. For example, the combination of threefold screw axis operation $6_{3}$ and inversion generate mirror reflection $M_{z}$ with the mirror plane contains an Mo triangle, which does not have the inversion center. It is the operator $\mathcal{A}=M_{z}$ that bunches the four states $\psi, P T \psi, P \psi$, and $T \psi$ together at the four TRI $k$ points in the $k_{z}=\pi$ plane, corresponding to one cubic DP and three linear DPs.

\section{Dirac fermions with different types of band dispersion}

Figure 2(a) illustrates the DFT-calculated band dispersion of $\mathrm{Tl}(\mathrm{MoTe})_{3}-\mathrm{a}$ representative of quasi-1D $\mathrm{A}(\mathrm{MoX})_{3}$ compounds that are immune to Peierls distortion. We find that the dispersion within either the $k_{z}=0$ $(\Gamma-\mathrm{M}-\mathrm{K}-\Gamma)$ or $k_{z}=\pi$ plane (A-H-L) is relatively flat. In contrast, the bands along the $c$ axis $(\Gamma-\mathrm{A}$ and $\Gamma-\mathrm{L})$ are dispersive, indicating the quasi-1D feature of the structure. Specifically, there are two steep and linear bands from conduction and valence bands meeting at the $\mathrm{A}(0,0,0.5)$ and $\mathrm{L}(0.5,0,0.5)$ points from $\Gamma$. Note that within the gap window of about $0.9 \mathrm{eV}$ around the Fermi level, only the Dirac bands show up within the Brillouin zone. Such a clean band structure is expected to be easy to capture by angle-resolved photoemission spectroscopy (ARPES) measurement. The Fermi velocity of the linear Dirac bands along the $k_{z}$ direction is $5.2 \times 10^{5} \mathrm{~m} / \mathrm{s}$, a value approaching that of graphene, indicating massless Dirac fermions with high mobility.

Next, we discuss the physical properties of the DPs located at the A and L points, respectively. There are four inequivalent DPs at the TRI $k$ points of the BZ boundary, including one $\mathrm{A}$ point and three $\mathrm{L}$ points, as indicated in Fig. 1(f). Here, the DPs at A and L are protected by the screw axis symmetry $2_{1}$ and are thus stable under adiabatic transformations that preserve these symmetry operations. To capture the dispersion physics of the DPs at A and L, we use a four-band $k \bullet p$ model applied at the high-symmetry points $\mathrm{A}$ and $\mathrm{L}$ and then confirm the results by DFT calculations. In this case, the matrix representation of the inversion symmetry operator $P$ has the form $P= \pm \tau_{x}$, while the time-reversal symmetry operator $T$ takes the form
$T=i \sigma_{y} K$, where $\boldsymbol{\tau}$ and $\boldsymbol{\sigma}$ represent the Pauli matrix working on the orbital and spin subspaces, respectively, and $K$ denotes complex conjugation. Taking the four-band basis $\{|\mathrm{A}, \uparrow\rangle,|\mathrm{B}, \uparrow\rangle,|\mathrm{A}, \downarrow\rangle,|\mathrm{B}, \downarrow\rangle\}$, with $\mathrm{A} / \mathrm{B}$ and $\uparrow / \downarrow$ denoting orbital and spin degrees of freedom, the low-energy Hamiltonian of the DP, with both $P$ - and $T$-invariance conditions implemented, is written in the form of a $4 \times 4$ matrix $[15,39]$ :
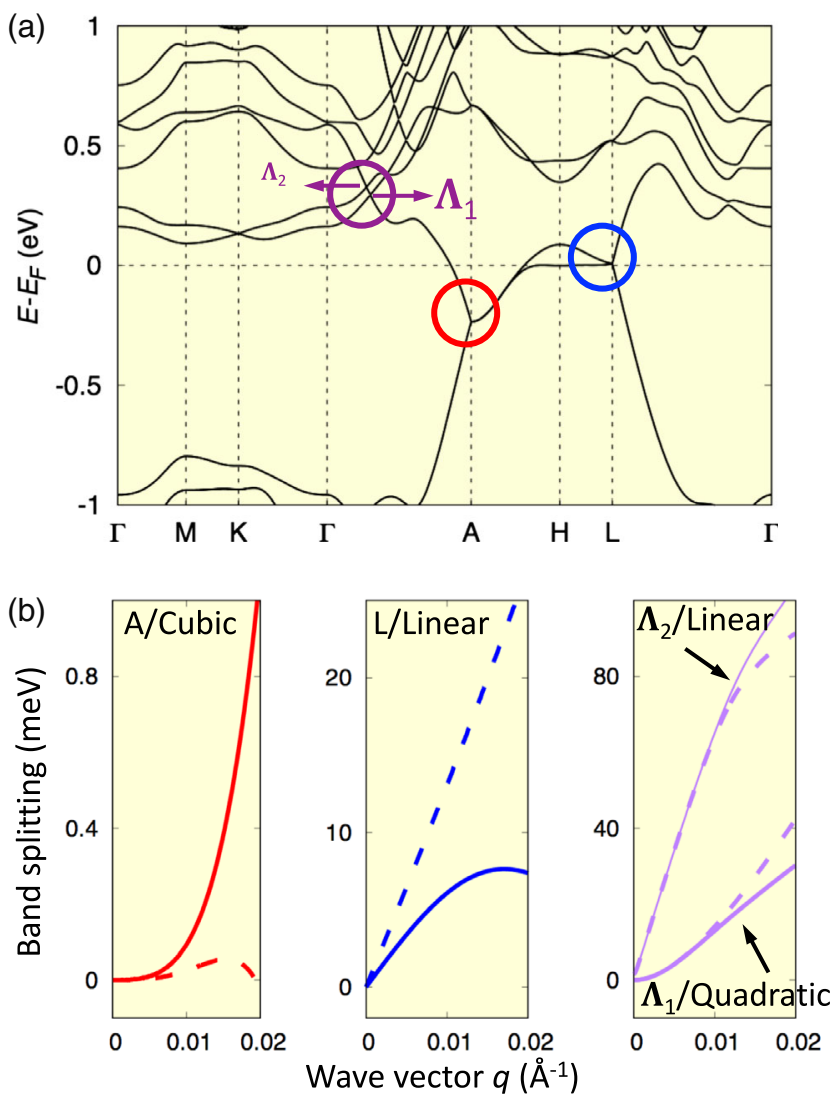

FIG. 2. (a) DFT calculated band structure of $\mathrm{Tl}(\mathrm{MoTe})_{3}$ as a representative of stable $\mathrm{A}(\mathrm{MoX})_{3}$ compounds. The different types of Dirac points are marked by red, blue, and purple circles. (b) In-plane band splitting in the vicinity of the four types of Dirac fermions at A, L, $\boldsymbol{\Lambda}_{1}$, and $\boldsymbol{\Lambda}_{2}$ points. The solid (dashed) lines denote DFT band splitting along the $k_{x}\left(k_{y}\right)$ direction, within the coordinate system defined in Fig. 1(f). 


$$
H(\boldsymbol{k})=\left(\begin{array}{cc}
a_{1}(\boldsymbol{k}) \sigma_{z}+a_{2}(\boldsymbol{k}) \sigma_{y}+a_{5}(\boldsymbol{k}) \sigma_{x} & \left(a_{3}(\boldsymbol{k})-i a_{4}(\boldsymbol{k})\right) \sigma_{z} \\
\left(a_{3}(\boldsymbol{k})+i a_{4}(\boldsymbol{k})\right) \sigma_{z} & -a_{1}(\boldsymbol{k}) \sigma_{z}+a_{2}(\boldsymbol{k}) \sigma_{y}+a_{5}(\boldsymbol{k}) \sigma_{x}
\end{array}\right),
$$

where $a_{0,5}(\boldsymbol{k})$ are real even functions of $\boldsymbol{k}$, and $a_{1,2,3,4}(\boldsymbol{k})$ are real odd functions of $\boldsymbol{k}$. By further applying specific rotation symmetries, the DPs could be classified according to their leading order of band dispersion based on different band eigenvalues of the rotation operator. Generally, the eigenvalues of $\mathrm{C}_{n}$ rotation are $\alpha=e^{i \pi(2 p+1) / n}$, where $p=0,1, \ldots, n-1$. If we use the $p$ value to represent the rotation eigenvalue of the four-band basis, we have a $\{p, q, r, s\}$ list, with the time-reversal symmetry condition requiring $r=n-p-1$ and $s=n-q-1$. Furthermore, the invariance of the Hamiltonian under $\mathrm{C}_{n}$ rotation forces all the $k$-expansion terms $k_{+}^{m_{1}} k_{-}^{m_{2}}$ in $H\left(k_{+}, k_{-}, k_{z}\right)$ to vanish if $m_{1}-m_{2} \neq p-q \bmod n$ (a more detailed derivation could be found in Refs. [7,15]). Therefore, the leading order of $k$ dispersion at the DP can be written as $p-q \bmod n$.

In $\mathrm{A}(\mathrm{MoX})_{3}$ compounds, the little group of the $\mathrm{A}$ point is $\mathrm{C}_{6 h}$ with a $\mathrm{C}_{6}$ rotation axis. Indeed, we find that the band eigenvalues of $\mathrm{C}_{6}$ at the $\mathrm{A}$ point are $\{i,-i,-i, i\}$, which means $\{p, q\}=4,1$ (the order does not matter). Therefore, the leading order of in-plane $k$ dispersion is 3 , indicating cubic Dirac fermions at A. It describes fermions having linear dispersion along the $\mathrm{C}_{6}$ axis ( $k_{z}$ direction), while they have cubic dispersion within the $k_{x}-k_{y}$ plane [the coordinate system is defined in Fig. 1(f)]. This result is confirmed by DFT calculations shown in Fig. 2(b), where it is clear that the band splitting between Dirac bands disperses cubically along both the $k_{x}$ and $k_{y}$ directions. The cubic Dirac fermion with isotropic dispersion is famous in the nonlinear self-interacting Dirac model in quantum field theory [40], while here we report an anisotropic cubic Dirac fermion in a real material for the first time. The effective Hamiltonian in the vicinity of the cubic DP is thus written as

$$
H_{\text {Cubic }}(\boldsymbol{k}) \sim\left(\begin{array}{cc}
v_{x}\left(k_{+}^{3}+k_{-}^{3}\right) \sigma_{x}+i v_{y}\left(k_{+}^{3}-k_{-}^{3}\right) \sigma_{y}+v_{z} k_{z} \sigma_{z} & 0 \\
0 & -v_{x}\left(k_{+}^{3}+k_{-}^{3}\right) \sigma_{x}-i v_{y}\left(k_{+}^{3}-k_{-}^{3}\right) \sigma_{y}-v_{z} k_{z} \sigma_{z}
\end{array}\right),
$$

where $k_{ \pm}=k_{x} \pm i k_{y}$, with the origin at the DP, and $v_{x, y, z}$ are independent real coefficients. Such a block-diagonal matrix can be decomposed into two Weyl Hamiltonians, with each one being characterized by a topological invariant, i.e., a Chern number, which is defined as the number of monopoles of Berry curvature of a closed 2D surface enclosing the Weyl nodes. In $\mathrm{A}(\mathrm{MoX})_{3}$, the $\mathrm{DP}$ at $\mathrm{A}$ is composed of two opposite cubic Weyl fermions [7] carrying Chern numbers +3 and -3 , joining without annihilation. In other words, such a DP can be viewed as being composed of six conventional Weyl fermions, with three having left-handed and three having right-handed chirality. It is known in high-energy physics that the Weyl fermions with the same chirality cannot be degenerate, so such a sixfold DP is indeed a "new fermion," caused by symmetries present specifically in crystals.

Next, we consider the L point, which has the little group $\mathrm{C}_{2 h}$ with a $\mathrm{C}_{2}$ rotation axis. Thus, the band eigenvalue of the rotation operator is $\pm i$, and $\{p, q\}=\{0,1\}$. Therefore, the $\mathrm{DP}$ at $\mathrm{L}$ has linear dispersion along the $k_{x}$ and $k_{y}$ directions, as confirmed by the DFT calculation shown in Fig. 2(b). The type of linear $\mathrm{DP}$ at $\mathrm{L}$ in $\mathrm{A}(\mathrm{MoX})_{3}$ is identical to that of hypothetical $\beta$-cristobalite $\mathrm{BiO}_{2}$ [11] and distorted spinel $\mathrm{BaZnSiO}_{4}$ [41], but it is more experimentally accessible because of its stability (as discussed in Sec. II. D) and the successful history of synthetization [24]. In addition, the DP at $\mathrm{L}$ in $\mathrm{Tl}(\mathrm{MoTe})_{3}$ is only $7 \mathrm{meV}$ below the Fermi level, which hopefully could be observed by ARPES.
The crystal symmetry of $\mathrm{A}(\mathrm{MoX})_{3}$ compounds can also host another type of Dirac fermion, which is induced by the band inversion between conduction and valence bands with an accidental band crossing inside the BZ. Examples include $\mathrm{Na}_{3} \mathrm{Bi}$ and $\mathrm{Cd}_{3} \mathrm{As}_{2}$, which were verified by ARPES measurement [13,14], and ternary honeycomb materials such as $\mathrm{BaYBi}(\mathrm{Y}=\mathrm{Au}, \mathrm{Ag}$, and $\mathrm{Cu}$ ) [42], as well as metastable allotropes of $\mathrm{Ge}$ and $\mathrm{Sn}$ [43] predicted by firstprinciples calculation. However, because the conduction and valence bands of the $\mathrm{A}(\mathrm{MoX})_{3}$ family only meet at $\mathrm{A}$ and $\mathrm{L}$ points, such DPs are actually band crossings of two conduction bands or two valence bands at $\boldsymbol{\Lambda}$ points along the $\Gamma$-A direction. $\mathrm{In} \mathrm{Tl}(\mathrm{MoTe})_{3}$, there are two inequivalent DPs that have energy of about $300 \mathrm{meV}$ above the Fermi level, as marked by the purple circles in Fig. 2(a). Interestingly, although they are located close to each other in terms of both momentum and energy, the dispersion properties of the two DPs are quite different. In contrast to the band crossings at $A$ and $L$ that are protected by nonsymmorphic symmetry, the DPs at $\boldsymbol{\Lambda}$ originate from the inversion of bands with different parities. Thus, the inversion operator takes the form $\mathrm{P}= \pm \tau_{z}$, and the low-energy Hamiltonian of the DP deviates from Eq. (1) accordingly [39]. Given the double group representations of $\mathrm{C}_{6}$, the twofold degenerate $\boldsymbol{\Lambda}$ bands have three possibilities: $\mathrm{G}_{7}+\mathrm{G}_{8}, \mathrm{G}_{9}+\mathrm{G}_{10}$, and $\mathrm{G}_{11}+\mathrm{G}_{12}$, with the eigenvalues of $\mathrm{C}_{6}$ being $e^{ \pm 5 \pi i / 6}, e^{ \pm \pi i / 6}$, and $e^{ \pm 3 \pi i / 6}$, respectively. For the DPs, $\Lambda_{1}$ is the crossing point of $\mathrm{G}_{7}+\mathrm{G}_{8}$ and 
$\mathrm{G}_{9}+\mathrm{G}_{10}$ bands, and thus, they have $\{p, q\}=\{2,0\}$, indicating a quadratic Dirac fermion with quadratic in-plane dispersion. While the quadratic Weyl points with the Chern numbers \pm 2 are theoretically predicted in time-reversal breaking $\mathrm{HgCrSe}_{4}$ [7] and inversion breaking $\mathrm{SrSi}_{2}$ [22], the quadratic Dirac fermion composed of two quadratic Weyl fermions with opposite monopole charges has never been reported before. On the other hand, $\boldsymbol{\Lambda}_{2}$ is the crossing point of $\mathrm{G}_{7}+\mathrm{G}_{8}$ and $\mathrm{G}_{11}+\mathrm{G}_{12}$ bands, and thus, we have $\{\mathrm{p}, \mathrm{q}\}=\{2,1\}$, indicating a linear Dirac fermion. We note that the linear DP at $\boldsymbol{\Lambda}_{2}$ can be distinguished from the DP at L in two ways: First, the DP at $\boldsymbol{\Lambda}_{2}$ originates from band crossing due to band inversion and happens in pairs around a TRI $k$ point [see Fig. 1(f)], while the DP at L is the touching point between the conduction and valence bands at the boundary of BZ due to nonsymmorphic symmetry; second, like A and $\boldsymbol{\Lambda}_{1}$, the DP at $\boldsymbol{\Lambda}_{2}$ shows isotropic dispersion between the $k_{x}$ and $k_{y}$ directions because the DPs along the $z$ axis feel $\mathrm{SO}(2)$ symmetry at small in-plane $\boldsymbol{k}$, while the DP at $\mathrm{L}$ has anisotropic linear dispersions along the $k_{x}$ and $k_{y}$ directions, as shown in Fig. 2(b). We summarize the physical characteristics of the different DPs in $\mathrm{Tl}(\mathrm{MoTe})_{3}$ in Table I.

\section{Peierls distortion and stable structures in quasi-1D $\mathrm{A}(\mathrm{MoX})_{3}$ compounds}

As noted above, the group of materials $\mathrm{A}(\mathrm{MoX})_{3}$ with reported $\mathrm{P}_{3} / \mathrm{m}$ structure are stable in the laboratory. However, the structure determination performed so far $[24,44]$ did not shed light on the possibility of possible Peierls distortion. It is natural to expect that the ideal 1D metallic structures are unstable against Peierls distortion and could have, in the undistorted structure, either soft phonon modes or higher energy than the distorted phase. Such distortion could destroy the crystal symmetries responsible for the DP and thus open a gap. To predict realistic quasi1D DSM candidates, we thus studied, systematically by DFT, the phonon spectra and thermodynamic stability of the $15 \mathrm{~A}(\mathrm{MoX})_{3}$ compounds related to Peierls distortion (shown in Fig. 3 and Appendix B). A number of observations can be made: (i) Four compounds $\mathrm{Na}(\mathrm{MoS})_{3}, \mathrm{Na}(\mathrm{MoSe})_{3}$, $\mathrm{K}(\mathrm{MoSe})_{3}$, and $\mathrm{Rb}(\mathrm{MoSe})_{3}$ have soft phonons for the undistorted structure, indicating dynamical instability (see Fig. 6). (ii) All six $\mathrm{A}(\mathrm{MoX})_{3}$ compounds with $\mathrm{A}=\mathrm{Na}, \mathrm{K}$, $\mathrm{Rb}$ and $\mathrm{X}=\mathrm{S}$ and $\mathrm{Se}$, including the four compounds having soft phonon modes, are highly unstable in the undistorted structure. As a result, the ground states of these materials experience Peierls transition to lower the total energy by 5.5-6.9 meV/f.u. and thus become semiconducting. (iii) $\mathrm{Tl}(\mathrm{MoTe})_{3}$ and $\mathrm{Rb}(\mathrm{MoTe})_{3}$ are immune to Peierls distortion, leading to the retention of the CDSM phase. (iv) There is an intermediate phase with seven materials. Their undistorted and distorted structures are both dynamically stable and have somewhat similar total energies, implying the coexistence of both phases.

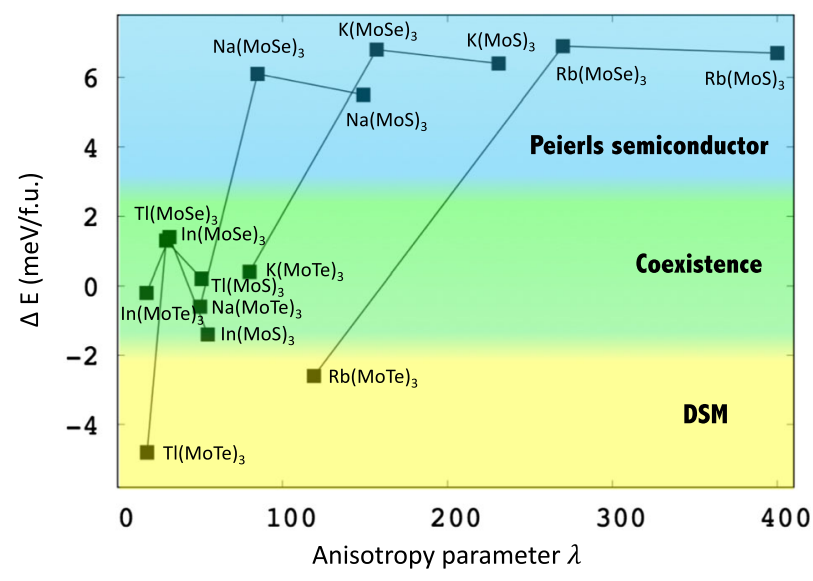

FIG. 3. (a) Phase diagram of DSM vs Peierls distortion as a function of anisotropy parameter $\lambda$ for $15 \mathrm{~A}(\mathrm{MoX})_{3}$ compounds. The black lines connecting compounds with the same A cation are a guide to the eye.

Several $\mathrm{A}(\mathrm{MoX})_{3}$ compounds are not dynamically stable in the high-symmetry $\mathrm{P}_{3} / \mathrm{m}$ structure, e.g., $\mathrm{K}(\mathrm{MoSe})_{3}$. The phonon spectrum of undistorted $\mathrm{K}(\mathrm{MoSe})_{3}$ is shown in Fig. 4(a). By analyzing the evolution of the phonon eigenvectors, we find that one soft phonon mode is found at $\Gamma$ as well as in the $k_{z}=0$ plane, indicating that such high-symmetry metallic structure is dynamically unstable. The eigenvectors of the soft phonon modes of undistorted $\mathrm{K}(\mathrm{MoSe}) 3$ [see Fig. 4(b)] show that Peierls distortion naturally happens. The Mo triangles tend to become airs by moving towards each other, forming alternative
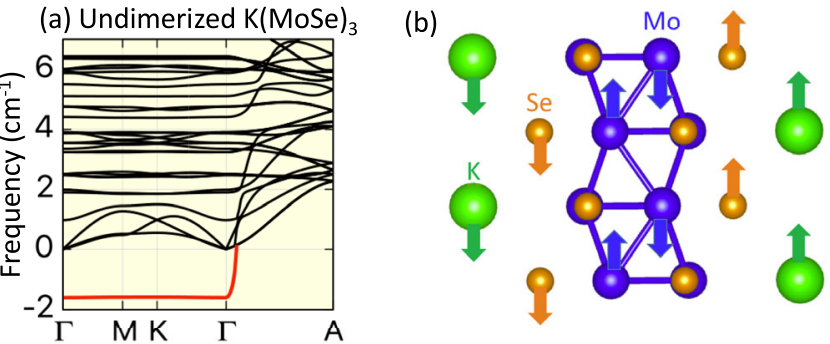

(c) Dimerized K(MoSe)
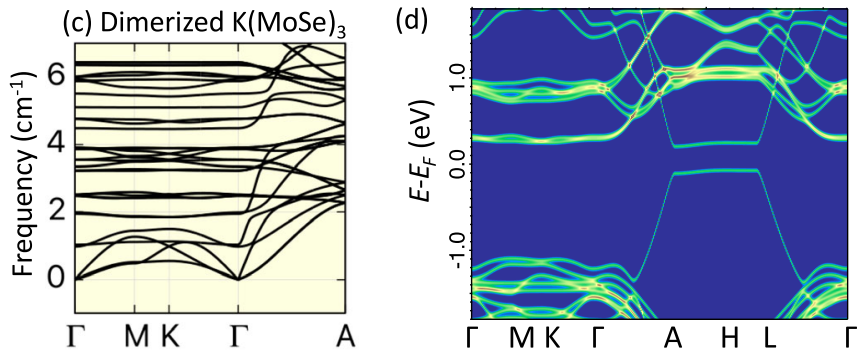

FIG. 4. Calculated phonon spectrum of undistorted $\mathrm{K}(\mathrm{MoSe})_{3}$. The red phonon dispersion indicates soft phonon modes. (b) Vibration modes of the soft phonon at the $\Gamma$ point in (a). (c) Calculated phonon spectrum of $\mathrm{K}(\mathrm{MoSe})_{3}$ under Peierls distortion with the soft mode eliminated. (d) Band dispersion of distorted $\mathrm{K}(\mathrm{MoSe})_{3}$ showing a band gap throughout the $k_{z}=\pi$ plane (A-H-L). 
short-long-short-long bonding with each other. Interestingly, the three Se atoms within the same plane of each Mo triangle tend to move oppositely and thus form a buckled in-plane structure. We applied such a distortion mode to the undistorted structure, and after relaxation, we found that such Peierls distortion indeed eliminates the negative phonon modes [see Fig. 4(c)] as well as lowers the total energy by $6.8 \mathrm{meV}$ per f.u. The distorted structure [see Fig. 1(d)] has a reduced symmetry with a space group of $\mathrm{P} \overline{3}$ (No. 147), in which the screw axis symmetry is no longer preserved. As a result, a band gap is opened at the $\mathrm{A}$ and $\mathrm{L}$ points. Figure 4(d) shows that in distorted $\mathrm{K}(\mathrm{MoSe})_{3}$, there is a $280-\mathrm{meV}$ band gap throughout the $k_{z}=\pi$ plane, indicating that the relative small change in band length between Mo triangles $(0.03 \AA)$ induces a remarkable effect in the electronic structure.

Indeed, the existence of Peierls distortion is basically the competition between band eigenvalues and elastic energy. If the gain of occupied band eigenvalues induced by creating a gap is less than the cost of elastic energy by modulating the atomic positions, Peierls distortion will not occur. The subtlety of whether a quasi-1D system would experience Peierls transition is closely related to how " $1 \mathrm{D}$ " the system is. To demonstrate this, we investigate the relationship between the stability of undistorted $\mathrm{A}(\mathrm{MoX})_{3}$ compounds and their character of one dimensionality. Since the linear Dirac bands along $\Gamma$-A are mainly contributed by $d_{x z}$ and $d_{y z}$ orbitals of an Mo atom, these states strongly extend along the chain direction through the intertriangle Mo-Mo bonding, leading to large dispersion. On the other hand, the energy bands within the $a-b$ plane are rather narrow, implying weak in-plane hopping. In particular, the flatness of the bands within the $k_{z}=\pi$ plane reflects the instability due to Fermi nesting. Therefore, we define a parameter $\lambda=W_{c} / W_{a b}$ to quantify the anisotropy between the $c$ axis and the $a-b$ plane, where $W_{c}$ and $W_{a b}$ denote the widths of the linear Dirac band along $\Gamma$-A and the flat band within the $k_{z}=\pi$ plane, to find out the relationship between Peierls distortion and the strength of the "1D-ness." More details about $\lambda$ for the $15 \mathrm{~A}(\mathrm{MoX})_{3}$ compounds are shown in Table III. The phase diagram as a function of $\lambda$ is shown in Fig. 3. A clear trend can be found: The compounds having a stable DMS phase or a competitive DSM phase compared to the Peierls phase are mostly located at the small $\lambda$ area, indicating that they are less 1D-like. On the other hand, the compounds with $\lambda>100$ are considered more 1D-like and are thus stabilized as a Peierls phase [except $\left.\mathrm{Rb}(\mathrm{MoTe})_{3}\right]$. In addition, for the group of $\mathrm{A}(\mathrm{MoX})_{3}$ with the same A cation, the $\mathrm{A}(\mathrm{MoSe})_{3}$ is the most unstable compared to $\mathrm{X}=\mathrm{S}$ and Te.

Finally, we predict that the quasi-1D feature of the $\mathrm{A}(\mathrm{MoX})_{3}$ family provides a platform for realizing low-dimensional physics as well as new electronic and optoelectronic device concepts. Specifically, the optical properties of DSM compounds are uniquely interesting
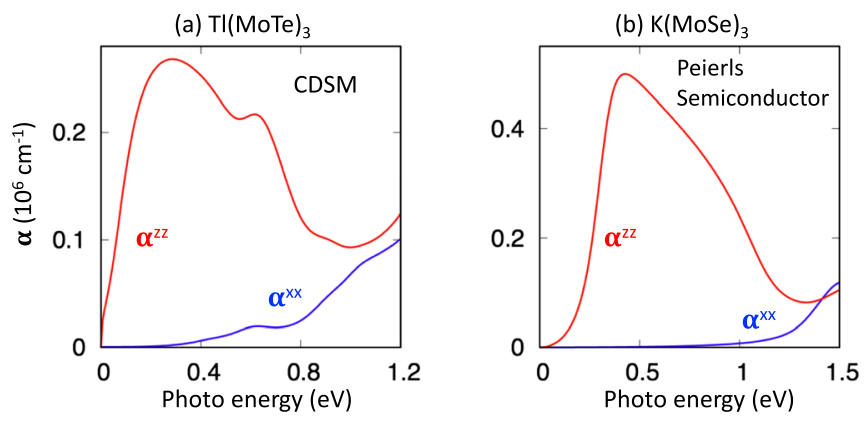

FIG. 5. Absorption coefficient $\left(\alpha^{x x}=\alpha^{y y} ; \quad \alpha^{z z}\right)$ of (a) $\mathrm{Tl}(\mathrm{MoTe})_{3}$ and (b) $\mathrm{K}(\mathrm{MoSe})_{3}$ from DFT calculations.

because of the coexistence of a reduced dimensionality 1D metal embedded in a semiconductor bulk, leading to a polarization-dependent optical signature. As shown in Fig. 5(a), for $\mathrm{Tl}(\mathrm{MoTe})_{3}$, there is an absorption peak starting at $0 \mathrm{eV}$ for $z$-polarized light originating from the interband optical transitions between the linearly dispersed valence and conduction Dirac bands, while there is nearly zero absorption for $x$ - and $y$-polarized light up to $0.5 \mathrm{eV}$, originating from the insulating states along these directions. The case of the Peierls semiconductor, e.g., $\mathrm{K}(\mathrm{MoSe})_{3}$, is slightly different in that the $z$ direction behaves like a smallgap semiconductor, evidenced by the nonzero energy onset of the absorption peak shown in Fig. 5(b). Future measurement of the polarization dependence will serve to disentangle the basically different optical properties of 1D metals and the semiconductor.

\section{DISCUSSION}

\section{A. Metal-insulator transition}

We expect that Peierls distortion is more noticeable at low temperatures, while at high temperatures, the highsymmetry metallic state is usually more stable because the gain of band eigenvalues is reduced by the thermal excitation of electrons across the band gap. Given that the structural determination of a single crystal assigns $\mathrm{A}(\mathrm{MoX})_{3}$ to the high-symmetry space group $\mathrm{P}_{3} / \mathrm{m}$ at high temperature, Fig. 3 could provide some implications on the metal-insulator transition of this system that can be compared with the transport measurements [28,29] and theoretical explanations using electron-phonon coupling [26]. We conclude that the compounds in the "Peierls semiconductor" region should undergo a phase transition from metal to semiconductor when the temperature goes below a critical value, while at low temperatures, the compounds in the "Coexistence" region could appear in both phases based on the growth condition. The phase diagram with three regions shown in Fig. 3 is in great agreement with the measurements made by previous experiments, including that $\mathrm{Tl}(\mathrm{MoTe})_{3}$ is metallic at low temperature; $\mathrm{A}(\mathrm{MoSe})_{3}$ compounds with $\mathrm{A}=\mathrm{Na}, \mathrm{K}, \mathrm{Rb}$ undergo a metal-insulator transition below $200 \mathrm{~K}$ [29]; 
and $\mathrm{Tl}(\mathrm{MoSe})_{3}$ and $\mathrm{In}(\mathrm{MoSe})_{3}$ have at least two types of samples, with one metallic down to low temperatures and another having a phase transition at a critical temperature [28]. Our results on thermodynamical stability are also consistent with the recent calculation using the electronphonon coupling, concluding that the main mechanism of the metal-insulator transition is the dynamic charge density wave that corresponds to Peierls-type displacement [26].

\section{B. Experimental accessibility}

The $\mathrm{A}(\mathrm{MoX})_{3}$ compounds were synthesized over 30 years ago [24] at $1000-1200{ }^{\circ} \mathrm{C}$ in sealed molybdenum crucibles under low argon pressure. The single crystals were needlelike growing along the $c$ axis, indicating the 1D character. Most of this family of compounds were found to be stable in air. It is noticeable that $\mathrm{Na}(\mathrm{MoSe})_{3}$, $\mathrm{In}(\mathrm{MoSe})_{3}$, and $\mathrm{Tl}(\mathrm{MoSe})_{3}$ were synthesized and reported to be quasi-1D superconductors with relatively high critical fields $[28,45,46]$. Some compounds from this family were shown to have metal-insulator transition below a critical temperature $[26,28,29]$. According to our DFT calculations, the compounds that do not have Peierls distortion and thus tend to stay metallic are $\mathrm{Tl}(\mathrm{MoTe})_{3}$ and $\mathrm{Rb}(\mathrm{MoTe})_{3}$. These tellurides are also predicted to have stronger dispersion than selenides and sulfides (see Fig. 7), providing a better chance to resolve the bands by ARPES.

Another challenge for detecting the cubic Dirac fermion is the possibility that the $\mathrm{A}$ cation in $\mathrm{A}(\mathrm{MoX})_{3}$ will show some off-stoichiometry, e.g., deficiency, which is a natural consequence of the high temperatures required in crystal growth. Indeed, single-crystal diffraction showed that while the structure of the quasi-1D MoX chain is nearly perfect, the A site shows up to $15 \%$ under stoichiometric [46]. One might be able to replenish the A-site deficiency on the surface by deposition of the cation ultrahigh vacuum evaporation so as to make the DP measurable by ARPES. Such a cation deficiency is expected to shift the Fermi energy downwards relative to the stoichiometric crystal, leading to unoccupied Dirac states that are not accessible to ARPES. Fortunately, the cubic DP of $\mathrm{Tl}(\mathrm{MoTe})_{3}$ is calculated to be $226 \mathrm{meV}$ below the Fermi level, so the downward shift may still place the DP near the Fermi energy.

\section{Remarks on surface states}

The link between the topological invariant of the bulk and the surface or edge states, known as bulk-boundary correspondence, is the central property of a topological system. For example, in WSMs, there are Fermi arcs robustly protected at the surface because of a topological origin [3]: Each 2D plane that lies between a pair of Weyl points, perpendicular to the separation between them, is a 2D Chern insulator associated with quantum Hall effect. The edge states of these Chern insulators connect to form surface Fermi arcs, which end at the projection of bulk
Weyl points. On the other hand, since the DP in DSM could be understood as two degenerate Weyl points with opposite monopole charge, one might expect that there are two copies of Fermi arcs on the DSM surface forming a ring with two singularities at the surface projection of the DPs in the bulk. However, a recent theoretical study reveals that the Fermi arcs on the DSM surface are not topologically protected and can be continuously deformed into the case of a topological or normal insulator without any symmetry breaking [47]. Then the surface behavior of a DSM will follow the direction where a small perturbation will lead the system to. For DSMs with a pair of DPs located away from the TRI $k$ points and $P= \pm \tau_{z}$ (parity inversion), such as $\mathrm{Na}_{3} \mathrm{Bi}$ and $\mathrm{Cd}_{3} \mathrm{As}_{2}$, a small perturbation can open the gap while preserving the band inversion, leading to a topological insulator (TI). Thus, the surface states are robust as a closed Fermi contour.

\section{Why are cubic Dirac fermions interesting relative to conventional Dirac fermions?}

DSMs with a DP located at the TRI $k$ points, such as $\mathrm{BiO}_{2}$ (in a hypothetical $\mathrm{SiO}_{2}$ structure [11]) and the $\mathrm{A}(\mathrm{MoX})_{3}$ family, behave as a quantum critical point, rendering it an ideal platform to realize other topological phases by symmetry tuning $[11,15,48]$. The main points of interest are as follows: (i) By breaking time-reversal symmetry in DSM, e.g., via the introduction of magnetic ions, such systems are known to transform to WSMs. More specifically, for CDSM, this was predicted theoretically [15] to lead to WSM with the unique occurrence of three Fermi arcs connecting the surface projection of the Weyl node and its antinode. This is the largest number of pairs of Weyl nodes that can theoretically be accommodated, leading to an enhanced conductivity step for the quantum anomalous Hall effect. (ii) The dispersion power $n$ (= numbers of monopole charges) present in symmetry-broken DSM was predicted to produce $n$-dependent quantum interference effects [49], leading to dispersion-dependent quantum transport phenomena. For conventional DSM or WSM $(n=1)$, a destructive quantum interference results in a weak antilocalization correction proportional to $-\sqrt{B}$ in the weak field limit. Such negative longitudinal magnetoresistance, also known as the chiral anomaly [50,51], has been confirmed by various transport measurements [52-56]. In contrast, for DSM or WSM with $n=2$, it has been predicted that a weak localization correction proportional to $+\sqrt{B}$ applies to the magnetoconductivity [49], calling for experimental verifications. With the material realization of $\operatorname{CDSM}(n=3)$, its transport behavior becomes an accessible and open question. (iii) The stronger screening of bare interaction and disorder in DSM or WSM with high-order dispersions provides the opportunity for more exotic physics, such as quantum criticality and phase transition [57-59]. In DSM or WSM, the density of states at the band-crossing point behaves as $\rho(E) \sim|E|^{2 / n}$. Compared with the 
linear-dispersing direction, the quadratic and cubicdispersing directions have enhanced density of states near the band-crossing point, which results in stronger screening. Specifically, in CDSM $(n=3)$, the Coulomb interactions along the in-plane directions are screened with a faster decay than that along the rotation axis $\left(r^{-1}\right)$. Recently, it was predicted that WSM with $n=3$, in the presence of shortrange interactions, can easily undergo a continuous quantum phase transition into either a translational symmetry-breaking axion insulator or a rotational symmetry-breaking nematic state [60]. Furthermore, the nonlinear dispersion and the 1D nature of a condensed-matter system would cause a breakdown of the interacting Fermi liquid theory for electron behavior, leading to Luttinger liquid instead.

\section{ACKNOWLEDGEMENTS}

We thank Professor L. Fu and Dr. J. Liu for reading this manuscript and making insightful comments. We also thank Professor V. Juricic, Dr. X. Zhang, Dr. M. Hoesch, and Dr. X. Zhou for helpful discussions and codes. This work was supported by the U.S. Department of Energy, Office of Science, Basic Energy Sciences, Materials Sciences and Engineering Division under Grant No. DE-SC0010467 to
University of Colorado, Boulder. This work used resources of the National Energy Research Scientific Computing Center, which is supported by the Office of Science of the U.S. Department of Energy under Contract No. DEAC02-05CH11231.

\section{APPENDIX A: CLASSIFICATION OF DIFFERENT TYPES OF FERMIONS IN SOLID-STATE PHYSICS}

Table II shows examples of materials that host different types of fermions classified by the degree of degeneracy $(g)$ and the highest power of band dispersion $(n)$. Here, we consider three-dimensional crystals with spin-orbit coupling, respecting time-reversal symmetry. We consider single-point degeneracy in $k$ space, so the materials with line nodes are not included. Some cases, e.g., $g=8$ and $n=3$, are forbidden because of the restriction of crystal symmetries. Some cases are predicted to exist, but there is no material realization yet (marked by "?" in the table). The materials with asterisks have hypothetical configurations, while the rest of the examples (including our work) have been synthesized as single crystals.

TABLE II. Different types of fermions in solid-state physics with example materials.

\begin{tabular}{|c|c|c|c|c|c|}
\hline \multirow[b]{2}{*}{$\mathrm{n}$} & \multicolumn{5}{|c|}{$\mathrm{g}$} \\
\hline & 2 (e.g., WSM) & 3 & 4 (e.g., DSM) & 6 & 8 \\
\hline 1 & TaAs [9], $\mathrm{WTe}_{2}$ [8] & $\mathrm{Pd}_{3} \mathrm{Bi}_{2} \mathrm{~S}_{2}$ [5], WC [19] & $\mathrm{Na}_{3} \mathrm{Bi}[4], \mathrm{BiO}_{2}{ }^{*}[11]$ & $\mathrm{MgPt}[5], \mathrm{Li}_{2} \mathrm{Pd}_{3} \mathrm{~B}$ [5] & $\mathrm{Ta}_{3} \mathrm{Sb}[5] \mathrm{Bi}_{2} \mathrm{AuO}_{5}$ [18] \\
\hline 2 & $\mathrm{SrSi}_{2}[22]$ & ZrTe [19], TaN [17] & $\alpha-\operatorname{Sn}[23]$ & $\mathrm{PdSb}_{2}[5]$ & Forbidden \\
\hline 3 & $?$ & Forbidden & $\mathrm{A}(\mathrm{MoX})_{3}$ (this work) & Forbidden & Forbidden \\
\hline
\end{tabular}

TABLE III. Calculated intertriangle Mo-Mo bond length $\left(d_{\text {int }}\right)$ of undistorted and distorted structures, $z$-direction Fermi velocity $v_{F}$ at the A point for undistorted structure, anisotropy parameter $\lambda$ for undistorted structure, and Peierls stabilization energy $\Delta E$ (the total energy difference between undistorted and distorted structures) for $15 \mathrm{~A}(\mathrm{MoX})_{3}$ compounds.

\begin{tabular}{|c|c|c|c|c|c|}
\hline Compounds & Undistorted $d_{\text {int }}(\AA)$ & Distorted $d_{\text {int }}(\AA)$ & $v_{F}(\mathrm{~m} / \mathrm{s})$ & $\lambda$ & $\Delta E$ (meV/f.u.) \\
\hline $\mathrm{Na}(\mathrm{MoS})_{3}{ }^{\mathrm{a}}$ & 2.690 & $2.666 / 2.714$ & $8.1 \times 10^{5}$ & 149 & 5.5 \\
\hline $\mathrm{Na}(\mathrm{MoSe})_{3}{ }^{\mathrm{a}}$ & 2.719 & $2.691 / 2.745$ & $8.3 \times 10^{5}$ & 84.8 & 6.1 \\
\hline $\mathrm{Na}(\mathrm{MoTe})_{3}$ & 2.762 & $2.730 / 2.801$ & $7.0 \times 10^{5}$ & 50 & -0.6 \\
\hline $\mathrm{K}(\mathrm{MoS})_{3}$ & 2.694 & $2.669 / 2.719$ & $8.4 \times 10^{5}$ & 231 & 6.4 \\
\hline $\mathrm{K}(\mathrm{MoSe})_{3}{ }^{\mathrm{a}}$ & 2.723 & $2.692 / 2.753$ & $8.0 \times 10^{5}$ & 157 & 6.8 \\
\hline $\mathrm{K}(\mathrm{MoTe})_{3}$ & 2.764 & $2.749 / 2.781$ & $7.3 \times 10^{5}$ & 80 & 0.4 \\
\hline $\mathrm{Rb}(\mathrm{MoS})_{3}$ & 2.696 & $2.672 / 2.720$ & $9.0 \times 10^{5}$ & 400 & 6.7 \\
\hline $\mathrm{Rb}(\mathrm{MoSe})_{3}{ }^{\mathrm{a}}$ & 2.724 & $2.696 / 2.753$ & $8.3 \times 10^{5}$ & 270 & 6.9 \\
\hline $\mathrm{Rb}(\mathrm{MoTe})_{3}$ & 2.765 & $2.763 / 2.768$ & $7.3 \times 10^{5}$ & 119 & -2.6 \\
\hline $\operatorname{In}(\mathrm{MoS})_{3}$ & 2.702 & $2.678 / 2.725$ & $8.2 \times 10^{5}$ & 54.6 & -1.4 \\
\hline $\mathrm{In}(\mathrm{MoSe})_{3}$ & 2.724 & $2.701 / 2.746$ & $6.9 \times 10^{5}$ & 31.4 & 1.3 \\
\hline $\mathrm{In}(\mathrm{MoTe})_{3}$ & 2.759 & $2.736 / 2.782$ & $5.4 \times 10^{5}$ & 17.5 & -0.2 \\
\hline $\mathrm{Tl}(\mathrm{MoS})_{3}$ & 2.699 & $2.676 / 2.722$ & $8.0 \times 10^{5}$ & 50.9 & 0.2 \\
\hline $\mathrm{Tl}(\mathrm{MoSe})_{3}$ & 2.723 & $2.701 / 2.746$ & $6.7 \times 10^{5}$ & 30.7 & 1.34 \\
\hline $\mathrm{Tl}(\mathrm{MoTe})_{3}$ & 2.760 & $2.735 / 2.785$ & $5.2 \times 10^{5}$ & 17.8 & -4.8 \\
\hline
\end{tabular}

${ }^{\mathrm{a}}$ Undistorted structure is dynamically unstable, i.e., has soft phonon modes. 


\section{APPENDIX B: FIRST-PRINCIPLES CALCULATIONS}

All calculations including total energy, electronic structure, and phonon dispersion were performed by density functional theory (DFT), where the geometrical and total energies are calculated by the projector-augmented wave (PAW) pseudopotential [61] and the exchange correlation is described by the generalized gradient approximation of Perdew, Burke, and Ernzerhof (PBE) [62] as implemented in the Vienna $a b$ initio package (VASP) [63]. The planewave energy cutoff is set to $500 \mathrm{eV}$, and the electronic energy minimization was performed with a tolerance of $10^{-5} \mathrm{eV}$. Spin-orbit coupling is taken into account selfconsistently throughout the electronic structure calculations. The atomic projection on band structure is calculated by projecting the wave functions with plan-wave expansion on the orbital basis (spherical harmonics) of each atomic site. The phonon spectra were identified using the PHONOPY package [64], in which the force constants are calculated in the framework of density-functional perturbation theory (DFPT) [65]. Phonon calculations were performed within a $1 \times 1 \times 4$ supercell (56 atoms). To evaluate the anisotropic
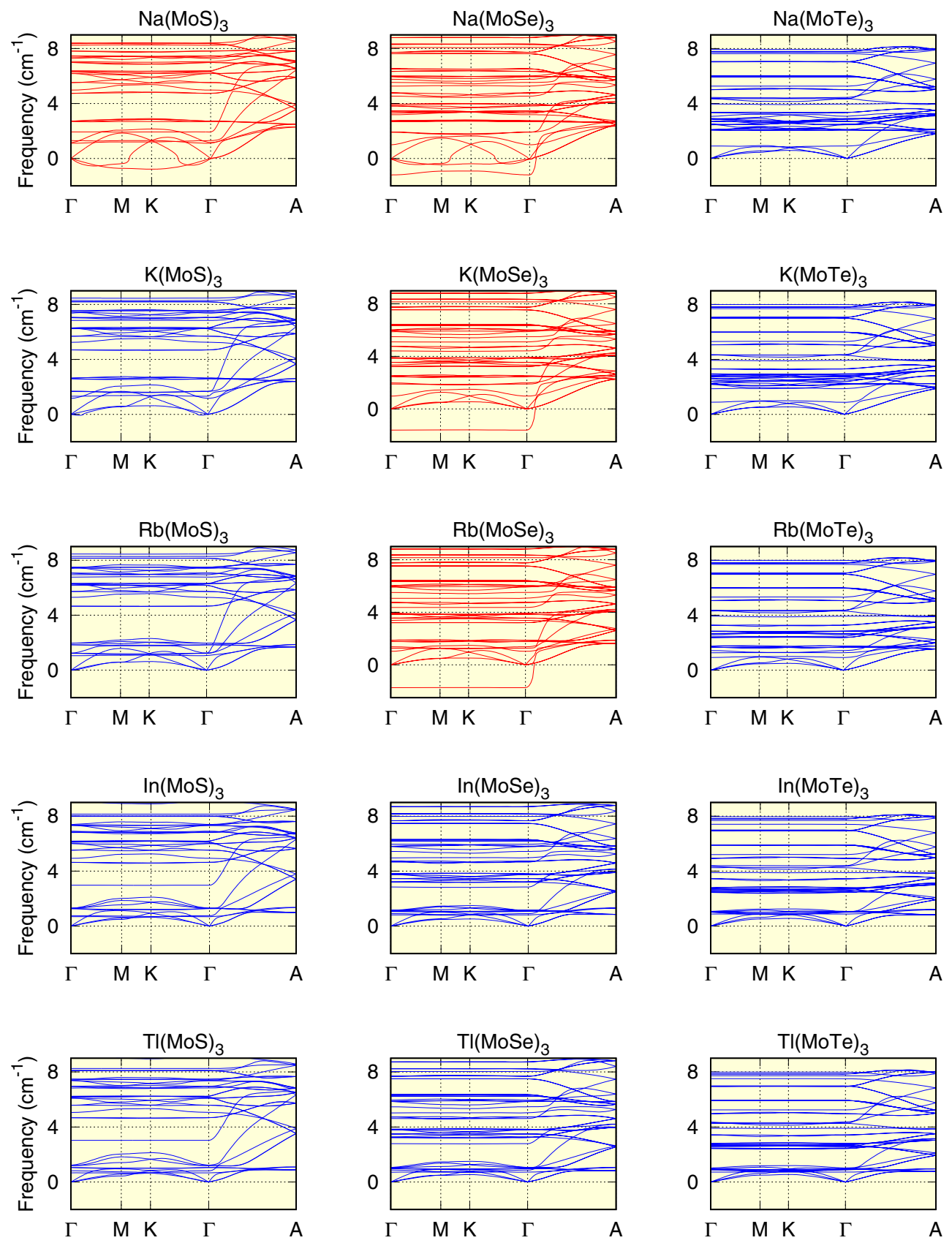

FIG. 6. Phonon spectra of $15 \mathrm{~A}(\mathrm{MoX})_{3}$ compounds with undistorted $\mathrm{P} 6_{3} / \mathrm{m}$ structure. The red and blue dispersions indicate spectra with soft phonons and without soft phonons, respectively. 
optical properties of quasi-1D $\mathrm{A}(\mathrm{MoX})_{3}$ compounds, we calculate the frequency $(\omega)$ dependent dielectric function $\epsilon^{i j}(\omega)$ based on DFT. Then, the optical absorption coefficient is evaluated from the dielectric function:

$$
\alpha^{i j}(\omega)=\frac{\sqrt{2} \omega}{c} \sqrt{\left|\epsilon^{i j}(\omega)\right|-\operatorname{Re}\left[\epsilon^{i j}(\omega)\right]},
$$

where $c$ is the speed of light and $\operatorname{Re}\left[\epsilon^{i j}(\omega)\right]$ is the real part of $\epsilon^{i j}(\omega)$.

In Table III, we show the geometry, electronic, and stability signatures of undistorted and distorted $\mathrm{A}(\mathrm{MoX})_{3}$ structures using DFT calculations. The results indicate that
9 out of the 15 compounds have comparative total energies for the two structures taken into consideration, and they are, in general, more 3D-like, as suggested by the anisotropy parameter $\lambda$.

Figure 6 shows the phonon spectra of $15 \mathrm{~A}(\mathrm{MoX})_{3}$ compounds with undistorted $\mathrm{P}_{3} / \mathrm{m}$ structure. We find that four compounds $\mathrm{Na}(\mathrm{MoS})_{3}, \mathrm{Na}(\mathrm{MoSe})_{3}, \mathrm{~K}(\mathrm{MoSe})_{3}$, and $\mathrm{Rb}(\mathrm{MoSe})_{3}$ are dynamically unstable because of the negative phonon modes for the $k_{z}=0$ plane of the undistorted $\mathrm{P}_{3} / \mathrm{m}$ structure.

Figure 7 shows the band structure of $15 \mathrm{~A}(\mathrm{MoX})_{3}$ compounds with undistorted $\mathrm{P}_{3} / \mathrm{m}$ structure. We find
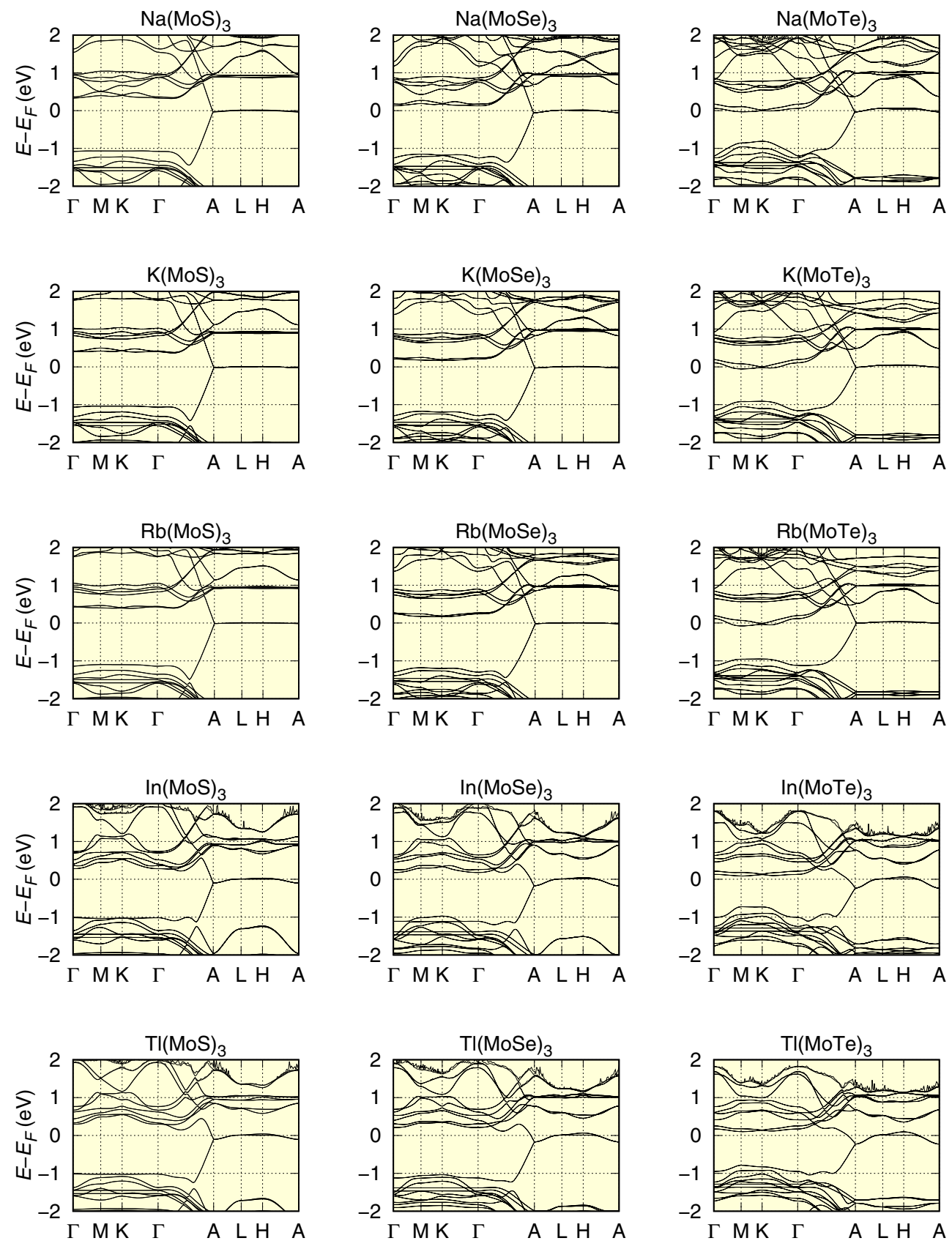

FIG. 7. Band structure of $15 \mathrm{~A}(\mathrm{MoX})_{3}$ compounds with undistorted $\mathrm{P} 6_{3} / \mathrm{m}$ structure. 
that all the quasi-1D compounds have strong dispersions along the $c$ axis and flat in-plane dispersions. Comparatively, the compounds with heavier $\mathrm{A}$ and $\mathrm{X}$ elements tend to have stronger in-plane dispersions because of the relativistic effects.

\section{APPENDIX C: SPACE GROUPS THAT HOST CUBIC DIRAC FERMIONS}

Here, we use symmetry analysis to show how nonsymmorphic symmetry ensures fourfold degeneracy, i.e., the Dirac point, at certain time-reversal invariant (TRI) $k$ points in a spin-orbit system preserving both inversion symmetry $P$ and time-reversal symmetry $T$. Then, we show that out of 230 space groups, only $\mathrm{P}_{3} / \mathrm{m}$ (No. 176) and $\mathrm{P} 6 /$ mcc (No. 192) have appropriate symmetries to host cubic Dirac fermions.

In a spin-orbit system, the anti-unitary operator $T$ behaves as $T^{2}=-1$, leading to Kramers degeneracy. Together with inversion symmetry, it turns out that all the energy bands are twofold degenerate with the two components related to each other by $P T$, i.e., $\psi(\boldsymbol{k}, \boldsymbol{\sigma})$ and $\operatorname{PT} \psi(\boldsymbol{k}, \boldsymbol{\sigma})=\psi(\boldsymbol{k},-\boldsymbol{\sigma})$, which is known as spin degeneracy. Therefore, to achieve fourfold degeneracy, we need an extra pair of states $L \psi$ and $P T L \psi$ with $[L, H]=0$ that differ with $\psi$ and $P T \psi$, while $L$ is a Hermitian symmetry operator of the system. We are thus looking for another Hermitian symmetry operator $\mathcal{A}$ to fulfill the condition $\left\{A, A_{P T}\right\} \cap\left\{A_{L}, A_{L P T}\right\}=\emptyset$, where $A$ is the eigenvalue of $\psi$ under $\mathcal{A}$. Thus, we get two pairs of bands $\{\psi, P T \psi\}$ and $\{L \psi, P T L \psi\}$ that have different eigenvalues of $\mathcal{A}$, so they must have a band crossing rather than a gap opening. The task is basically to find the two operators $L$ and $\mathcal{A}$, and the degeneracy will happen at the $k$ points that are invariant under these two symmetry operations. For example, if the $k$ points that are invariant under both $L$ and $\mathcal{A}$ form a line, the system is thus a nodal-line semimetal.

Without adding new symmetries, we first let $L=P$. Since the $P$ operator reverses the momentum, there are only eight TRI $k$ points in the $\mathrm{BZ}$ that are $P$ invariant. Now, we are looking for the operator $\mathcal{A}$ that fulfills

$$
\left\{A, A_{P T}\right\} \cap\left\{A_{P}, A_{T}\right\}=\emptyset .
$$

We next consider the most common twofold symmetries for $\mathcal{A}$ that all the TRI $k$ points can preserve, which have two eigenvalues. From Eq. (C1), we have $A=-A_{P}$, indicating

$$
\mathcal{A} P \psi=A_{P} P \psi=-P A \psi=-P \mathcal{A} \psi,
$$

which leads to the anticommutation relationship

$$
\{\mathcal{A}, P\}=0 .
$$

Given that $P$ commutes with any point-group operations, we conclude that $\mathcal{A}$ contains a nonsymmorphic symmetry that is a combination of point-group operation and fractional translation. In addition, from Eq. (1), there is another condition $A=-A_{T}$. Considering $[\mathcal{A}, \mathrm{T}]=0$, we have

$$
\mathcal{A} T \psi=T \mathcal{A} \psi=T A \psi=-T A_{T} \psi=A_{T} T \psi,
$$

which indicates $A_{T}= \pm i$ and thus

$$
\mathcal{A}^{2}=-1
$$

Therefore, the symmetry operation $\mathcal{A}$ that fulfills Eqs. (C3) and (C5) ensures a DP in certain TRI $k$ points.

Combining three symmetry filters for cubic Dirac semimetal (i.e., inversion, $\mathrm{C}_{6}$, and nonsymmorphic symmetry), only four possibilities $\left[\mathrm{P6}_{3} / \mathrm{m}\right.$ (No. 176), $\mathrm{P} 6 / \mathrm{mcc}$ (No. 192), $\mathrm{P}_{3} / \mathrm{mcm}$ (No. 193), and $\mathrm{P}_{3} / \mathrm{mmc}$ (No. 194)] are left. All of these space groups have DPs at the four TRI $k$ points (one A point and three L points) within the $k_{z}=\pi$ plane. For space groups No. 176, No. 193, and No. 194, there is an axis symmetry $\left\{\mathrm{C}_{2} \mid(0,0,1 / 2)\right\}$, which transforms $(x, y, z)$ in position space to $(-x,-y, z+1 / 2)$. Considering the combination symmetry $\mathcal{A}=P\left\{\mathrm{C}_{2} \mid(0,0,1 / 2)\right\}$, it is easy to test that $[\mathcal{A}, P]=0$ in the $k_{z}=0$ plane and $\{\mathcal{A}, P\}=0$ in the $k_{z}=\pi$ plane. On the other hand, $\mathcal{A}^{2}$ preserves $(x, y, z)$ while it rotates spin by $2 \pi$, leading to a minus sign, $\mathcal{A}^{2}=-1$. Therefore, $\mathcal{A}$ protects the fourfold degeneracy at the four TRI $k$ points within the $k_{z}=\pi$ plane. However, space groups No. 193 and No. 194 have three mirror planes parallel to the $\mathrm{C}_{6}$ axis, posing extra symmetry conditions that force three high-symmetry lines to be degenerate. Here, we still take $\mathcal{A}=P\left\{\mathrm{C}_{2} \mid(0,0,1 / 2)\right\}$ but $L=M_{x}$, which transforms $(x, y, z)$ in position space to $(-x, y, z)$. The commutation relationship then reads $\left\{\mathcal{A}, M_{x}\right\}=0$ and $\left[\mathcal{A}, P T M_{x}\right]=0$ in the $k_{z}=\pi$ plane. In this case, $\mathcal{A}$ and $L$ keep the whole $k_{x}=0$ line, as well as another two lines related by $\mathrm{C}_{3}$ symmetry in the $k_{z}=\pi$ plane, rendering the system a nodal-line or nodal-ring semimetal.

On the other hand, space group P6/mcc (No. 192) has six glide reflection planes that all contain the $\mathrm{C}_{6}$ axis, and here we take $\left\{M_{x} \mid(0,0,1 / 2)\right\}$, which transforms $(x, y, z)$ to $(-x, y, z+1 / 2)$. Similarly, considering the combination symmetry $\mathcal{A}=P\left\{M_{x} \mid(0,0,1 / 2)\right\}$, we also have $\{\mathcal{A}, P\}=$ 0 in the $k_{z}=\pi$ plane and $\mathcal{A}^{2}=-1$, which protects only four DPs and no extra symmetries for more degenerate $k$ points. Finally, we reach the conclusion that out of 230 space groups, only $\mathrm{P}_{3} / \mathrm{m}$ (No. 176) and P6/mcc (No. 192) have appropriate symmetries to host cubic Dirac fermions.

[1] C. J. Bradley and A. P. Cracknell, The Mathematical Theory of Symmetry in Solids (Clarendon, Oxford, 1972).

[2] M.S. Dresselhaus, G. Dresselhaus, and A. Jorio, Group Theory: Application to the Physics of Condensed Matter (Springer, New York, 2008). 
[3] X. Wan, A. M. Turner, A. Vishwanath, and S. Y. Savrasov, Topological Semimetal and Fermi-Arc Surface States in the Electronic Structure of Pyrochlore Iridates, Phys. Rev. B 83, 205101 (2011).

[4] Z. Wang, Y. Sun, X.-Q. Chen, C. Franchini, G. Xu, H. Weng, X. Dai, and Z. Fang, Dirac Semimetal and Topological Phase Transitions in $A_{3} B i(A=\mathrm{Na}, K, R b)$, Phys. Rev. B 85, 195320 (2012).

[5] B. Bradlyn, J. Cano, Z. Wang, M. G. Vergniory, C. Felser, R. J. Cava, and B. A. Bernevig, Beyond Dirac and Weyl Fermions: Unconventional Quasiparticles in Conventional Crystals, Science 353, 5037 (2016).

[6] G. Xu, H. Weng, Z. Wang, X. Dai, and Z. Fang, Chern Semimetal and the Quantized Anomalous Hall Effect in $\mathrm{HgCr}_{2} \mathrm{Se}_{4}$, Phys. Rev. Lett. 107, 186806 (2011).

[7] C. Fang, M. J. Gilbert, X. Dai, and B. A. Bernevig, MultiWeyl Topological Semimetals Stabilized by Point Group Symmetry, Phys. Rev. Lett. 108, 266802 (2012).

[8] A. A. Soluyanov, D. Gresch, Z. Wang, Q. Wu, M. Troyer, X. Dai, and B. A. Bernevig, Type-II Weyl Semimetals, Nature (London) 527, 495 (2015).

[9] H. Weng, C. Fang, Z. Fang, B. A. Bernevig, and X. Dai, Weyl Semimetal Phase in Noncentrosymmetric TransitionMetal Monophosphides, Phys. Rev. X 5, 011029 (2015).

[10] S.-Y. Xu, I. Belopolski, N. Alidoust, M. Neupane, G. Bian, C. Zhang, R. Sankar, G. Chang, Z. Yuan, C.-C. Lee et al., Discovery of a Weyl Fermion Semimetal and Topological Fermi Arcs, Science 349, 613 (2015).

[11] S. M. Young, S. Zaheer, J. C. Y. Teo, C. L. Kane, E. J. Mele, and A. M. Rappe, Dirac Semimetal in Three Dimensions, Phys. Rev. Lett. 108, 140405 (2012).

[12] Z. Wang, H. Weng, Q. Wu, X. Dai, and Z. Fang, ThreeDimensional Dirac Semimetal and Quantum Transport in $\mathrm{Cd}_{3} \mathrm{As}_{2}$, Phys. Rev. B 88, 125427 (2013).

[13] Z. K. Liu, J. Jiang, B. Zhou, Z. J. Wang, Y. Zhang, H. M. Weng, D. Prabhakaran, S. K. Mo, H. Peng, P. Dudin et al., A Stable Three-Dimensional Topological Dirac Semimetal $\mathrm{Cd}_{3} \mathrm{As}_{2}$, Nat. Mater. 13, 677 (2014).

[14] Z. K. Liu, B. Zhou, Y. Zhang, Z. J. Wang, H. M. Weng, D. Prabhakaran, S. K. Mo, Z. X. Shen, Z. Fang, X. Dai, Z. Hussain, and Y. L. Chen, Discovery of a Three-Dimensional Topological Dirac Semimetal, $\mathrm{Na}_{3} \mathrm{Bi}$, Science 343, 864 (2014).

[15] B.-J. Yang and N. Nagaosa, Classification of Stable ThreeDimensional Dirac Semimetals with Nontrivial Topology, Nat. Commun. 5, 4898 (2014).

[16] S. M. Young and C. L. Kane, Dirac Semimetals in Two Dimensions, Phys. Rev. Lett. 115, 126803 (2015).

[17] H. Weng, C. Fang, Z. Fang, and X. Dai, Topological Semimetals with Triply Degenerate Nodal Points in $\theta$-Phase Tantalum Nitride, Phys. Rev. B 93, 241202 (2016).

[18] B. J. Wieder, Y. Kim, A. M. Rappe, and C. L. Kane, Double Dirac Semimetals in Three Dimensions, Phys. Rev. Lett. 116, 186402 (2016).

[19] Z. Zhu, G. W. Winkler, Q. Wu, J. Li, and A. A. Soluyanov, Triple Point Topological Metals, Phys. Rev. X 6, 031003 (2016).

[20] T. T. Heikkilä and G. E. Volovik, Fermions with Cubic and Quartic Spectrum, JETP Lett. 92, 681 (2010).
[21] Z. Gao, M. Hua, H. Zhang, and X. Zhang, Classification of Stable Dirac and Weyl Semimetals with Reflection and Rotational Symmetry, Phys. Rev. B 93, 205109 (2016).

[22] S.-M. Huang, S.-Y. Xu, I. Belopolski, C.-C. Lee, G. Chang, T.-R. Chang, B. Wang, N. Alidoust, G. Bian, M. Neupane et al., New Type of Weyl Semimetal with Quadratic Double Weyl Fermions, Proc. Natl. Acad. Sci. U.S.A. 113, 1180 (2016).

[23] S. Groves and W. Paul, Band Structure of Gray Tin, Phys. Rev. Lett. 11, 194 (1963).

[24] M. Potel, R. Chevrel, M. Sergent, J. C. Armici, M. Decroux, and $\varnothing$. Fischer, New Pseudo-One-Dimensional Metals: $\mathrm{M}_{2} \mathrm{Mo}_{6} \mathrm{~S}_{6}(\mathrm{M}=\mathrm{Na}, I n, K, T I), \mathrm{M}_{2} \mathrm{Mo}_{6} \mathrm{~S}_{6}(\mathrm{M}=\mathrm{K}, R b$, Cs), $\mathrm{M}_{2} \mathrm{Mo}_{6} \mathrm{Te}_{6}(\mathrm{M}=\mathrm{In}, \mathrm{TI})$, J. Solid State Chem. 35, 286 (1980).

[25] T. Hughbanks and R. Hoffmann, Molybdenum Chalcogenides: Clusters, Chains, and Extended Solids. The Approach to Bonding in Three Dimensions, J. Am. Chem. Soc. 105, 1150 (1983).

[26] A. P. Petrovic, R. Lortz, G. Santi, M. Decroux, H. Monnard, $\emptyset$. Fischer, L. Boeri, O. K. Andersen, J. Kortus, D. Salloum, P. Gougeon, and M. Potel, Phonon Mode Spectroscopy, Electron-Phonon Coupling, and the Metal-Insulator Transition in Quasi-One-Dimensional $\mathrm{M}_{2} \mathrm{Mo}_{6} \mathrm{~S}_{6}$, Phys. Rev. B 82, 235128 (2010).

[27] Q. D. Gibson, L. M. Schoop, L. Muechler, L. S. Xie, M. Hirschberger, N. P. Ong, R. Car, and R. J. Cava, ThreeDimensional Dirac Semimetals: Design Principles and Predictions of New Materials, Phys. Rev. B 91, 205128 (2015).

[28] J. C. Armici, M. Decroux, Ø. Fischer, M. Potel, R. Chevrel, and M. Sergent, A New Pseudo-One-Dimensional Superconductor: $\mathrm{Tl}_{2} \mathrm{Mo}_{6} \mathrm{~S}_{6}$, Solid State Commun. 33, 607 (1980).

[29] J. M. Tarascon, F. J. DiSalvo, and J. V. Waszczak, Physical Properties of Several $\mathrm{M}_{2} \mathrm{Mo}_{6} \mathrm{X}_{6}$ Compounds $(\mathrm{M}=$ GROUP IA METAL; $\mathrm{X}=\mathrm{Se}, \mathrm{Te})$, Solid State Commun. 52, 227 (1984).

[30] Q.-F. Liang, J. Zhou, R. Yu, Z. Wang, and H. Weng, NodeSurface and Node-Line Fermions from Nonsymmorphic Lattice Symmetries, Phys. Rev. B 93, 085427 (2016).

[31] A. A. Burkov, M. D. Hook, and L. Balents, Topological Nodal Semimetals, Phys. Rev. B 84, 235126 (2011).

[32] C. Fang, Y. Chen, H.-Y. Kee, and L. Fu, Topological Nodal Line Semimetals with and without Spin-Orbital Coupling, Phys. Rev. B 92, 081201 (2015).

[33] H. Weng, Y. Liang, Q. Xu, R. Yu, Z. Fang, X. Dai, and Y. Kawazoe, Topological Node-Line Semimetal in ThreeDimensional Graphene Networks, Phys. Rev. B 92, 045108 (2015).

[34] R. Yu, H. Weng, Z. Fang, X. Dai, and X. Hu, Topological Node-Line Semimetal, and Dirac Semimetal State in Antiperovskite $\mathrm{Cu}_{3} \mathrm{PdN}$, Phys. Rev. Lett. 115, 036807 (2015).

[35] G. Bian, T.-R. Chang, R. Sankar, S.-Y. Xu, H. Zheng, T. Neupert, C.-K. Chiu, S.-M. Huang, G. Chang, I. Belopolski et al., Topological Nodal-Line Fermions in Spin-Orbit Metal PbTaSe 2 , Nat. Commun. 7, 10556 (2016).

[36] H. Huang, J. Liu, D. Vanderbilt, and W. Duan, Topological Nodal-Line Semimetals in Alkaline-Earth Stannides, 
Germanides, and Silicides, Phys. Rev. B 93, 201114 (2016).

[37] T. Bzdusek, Q. Wu, A. Rüegg, M. Sigrist, and A. A. Soluyanov, Nodal-Chain Metals, Nature, advance online publication (2016).

[38] R. Chevrel, P. Gougeon, M. Potel, and M. Sergent, Symposium on Metal-Metal Bonding in Solid State Clusters and Extended Arrays. Ternary Molybdenum Chalcogenides: A Route to New Extended Clusters, J. Solid State Chem. 57, 25 (1985).

[39] M. Shuichi, Phase Transition between the Quantum Spin Hall and Insulator Phases in 3D: Emergence of a Topological Gapless Phase, New J. Phys. 9, 356 (2007).

[40] F. W. Hehl and B. K. Datta, Nonlinear Spinor Equation and Asymmetric Connection in General Relativity, J. Math. Phys. (N.Y.) 12, 1334 (1971).

[41] J. A. Steinberg, S. M. Young, S. Zaheer, C. L. Kane, E. J. Mele, and A. M. Rappe, Bulk Dirac Points in Distorted Spinels, Phys. Rev. Lett. 112, 036403 (2014).

[42] Y. Du, B. Wan, D. Wang, L. Sheng, C.-G. Duan, and X. Wan, Dirac and Weyl Semimetal in XYBi $(\mathrm{X}=\mathrm{Ba}, E u$; $\mathrm{Y}=\mathrm{Cu}, A g$ and $A u$ ), Sci. Rep. 5, 14423 (2015).

[43] W. Cao, P. Tang, S.-C. Zhang, W. Duan, and A. Rubio, Stable Dirac Semimetal in the Allotropes of Group-IV Elements, Phys. Rev. B 93, 241117 (2016).

[44] R. Chevrel and M. Sergent, in Crystal Chemistry and Properties of Materials with Quasi-One-Dimensional Structures: A Chemical and Physical Synthetic Approach, edited by J. Rouxel (Springer Netherlands, Dordrecht, 1986), p. 315.

[45] R. Brusetti, P. Monceau, M. Potel, P. Gougeon, and M. Sergent, The Exotic Superconductor $\mathrm{Tl}_{2} \mathrm{Mo}_{6} \mathrm{~S}_{6}$ Investigated by Low Field Magnetization Measurements, Solid State Commun. 66, 181 (1988).

[46] A. P. Petrovic, D. Ansermet, D. Chernyshov, M. Hoesch, D. Salloum, P. Gougeon, M. Potel, L. Boeri, and C. Panagopoulos, A Disorder-Enhanced Quasi-One-Dimensional Superconductor, Nat. Commun. 7, 12262 (2016).

[47] M. Kargarian, M. Randeria, and Y.-M. Lu, Are the Surface Fermi Arcs in Dirac Semimetals Topologically Protected?, Proc. Natl. Acad. Sci. U.S.A. 113, 8648 (2016).

[48] H. Weng, X. Dai, and Z. Fang, Topological Semimetals Predicted from First-Principles Calculations, J. Phys. Condens. Matter 28, 303001 (2016).

[49] X. Dai, H.-Z. Lu, S.-Q. Shen, and H. Yao, Detecting Monopole Charge in Weyl Semimetals via Quantum Interference Transport, Phys. Rev. B 93, 161110 (2016).

[50] H. B. Nielsen and M. Ninomiya, The Adler-Bell-Jackiw Anomaly and Weyl Fermions in a Crystal, Phys. Lett. B 130, 389 (1983).
[51] A. A. Burkov, Negative Longitudinal Magnetoresistance in Dirac and Weyl Metals,Phys. Rev. B 91, 245157 (2015).

[52] C. Shekhar, A. K. Nayak, Y. Sun, M. Schmidt, M. Nicklas, I. Leermakers, U. Zeitler, Y. Skourski, J. Wosnitza, Z. Liu et al., Extremely Large Magnetoresistance and Ultrahigh Mobility in the Topological Weyl Semimetal Candidate NbP, Nat. Phys. 11, 645 (2015).

[53] C.-L. Zhang, S.-Y. Xu, I. Belopolski, Z. Yuan, Z. Lin, B. Tong, G. Bian, N. Alidoust, C.-C. Lee, S.-M. Huang et al., Signatures of the Adler-Bell-Jackiw Chiral Anomaly in a Weyl Fermion Semimetal, Nat. Commun. 7, 10735 (2016).

[54] X. Huang, L. Zhao, Y. Long, P. Wang, D. Chen, Z. Yang, H. Liang, M. Xue, H. Weng, Z. Fang, X. Dai, and G. Chen, Observation of the Chiral-Anomaly-Induced Negative Magnetoresistance in $3 D$ Weyl Semimetal TaAs, Phys. Rev. X 5 , 031023 (2015).

[55] H.-Z. Lu and S.-Q. Shen, Weak Antilocalization and Localization in Disordered and Interacting Weyl Semimetals, Phys. Rev. B 92, 035203 (2015).

[56] J. Xiong, S. K. Kushwaha, T. Liang, J. W. Krizan, M. Hirschberger, W. Wang, R. J. Cava, and N. P. Ong, Evidence for the Chiral Anomaly in the Dirac Semimetal $\mathrm{Na}_{3} \mathrm{Bi}$, Science 350, 413 (2015).

[57] H.-H. Lai, Correlation Effects in Double-Weyl Semimetals, Phys. Rev. B 91, 235131 (2015).

[58] S.-K. Jian and H. Yao, Correlated Double-Weyl Semimetals with Coulomb Interactions: Possible Applications to $\mathrm{HgCr}_{2} \mathrm{Se}_{4}$ and $\mathrm{SrSi}_{2}$, Phys. Rev. B 92, 045121 (2015).

[59] S.-K. Jian and H. Yao, Fermion-Induced Quantum Critical Points in 3D Weyl Semimetals, arXiv:1609.06313.

[60] B. Roy, P. Goswami, and V. Juricic, Interacting Weyl Fermions: Phases, Phase Transitions and Global Phase Diagram, arXiv:1610.05762.

[61] G. Kresse and D. Joubert, From Ultrasoft Pseudopotentials to the Projector Augmented-Wave Method, Phys. Rev. B 59, 1758 (1999).

[62] J. P. Perdew, K. Burke, and M. Ernzerhof, Generalized Gradient Approximation Made Simple, Phys. Rev. Lett. 77, 3865 (1996).

[63] G. Kresse and J. Furthmüller, Efficiency of Ab-Initio Total Energy Calculations for Metals and Semiconductors Using a Plane-Wave Basis Set, Comput. Mater. Sci. 6, 15 (1996).

[64] A. Togo and I. Tanaka, First Principles Phonon Calculations in Materials Science, Scr. Mater. 108, 1 (2015).

[65] X. Gonze and C. Lee, Dynamical Matrices, Born Effective Charges, Dielectric Permittivity Tensors, and Interatomic Force Constants from Density-Functional Perturbation Theory, Phys. Rev. B 55, 10355 (1997). 